\title{
Observer design and practical stability of nonlinear systems under unknown time-delay
}

\author{
Nadhem ECHI \\ Gafsa University, Faculty of Sciences of Gafsa \\ Department of Mathematics, Zarroug Gafsa 2112 Tunisia \\ E-mail: nadhemechi_fsg@yahoo.fr
}

\begin{abstract}
In the present paper, we study observer design and we establish some sufficient conditions for practical exponential stability for a class of time-delay nonlinear systems written in triangular form. In case of delay, the exponential convergence of the observer was confirmed. Based on the Lyapunov-Krasovskii functionals, the practical stability of the proposed observer is achieved. Finally, a physical model and simulation findings show the feasibility of the suggested strategy.
\end{abstract}

Mathematics Subject Classification. 93C10, 93D15, 93D20.

Keywords. Observer; exponential stability; practical stability; time delay; Lyapunov-Krasovskii.

\section{Introduction}

Time-delay systems are one of the basic mathematical models of real phenomena such as nuclear reactors, chemical engineering systems, biological systems [16, and population dynamics models [18]. The analysis of systems without delays is generally simple as compared to nonlinear systems under time delays. However, there are a number of problems relating to nonlinear observer for time delay system. In particular, a problem of theoretical and practical importance is the design of observer-based for time-delay systems. In literature, a separation principle, for nonlinear free-delay systems, using high gain observers is provided in [1] and [2]. Much attention has been paid to solve such a problem and many observer designs for time-delay nonlinear systems approaches have been used. In [26], it is shown that observer design for a class of nonlinear time delay systems is solved by using linear matrix inequality. In 11, some sufficient conditions for practical uniform stability of a class of uncertain time-varying systems with a bounded time-varying state delay were provided using the Lyapunov stability theory. 4] and 9] show that state and output feedback controllers of time-delay systems, written in a triangular linear growth condition are reached under delay independent conditions and under delay dependent conditions respectively.

The observer design problem for nonlinear systems satisfying a Lipschitz continuity condition has been a topic of numerous papers, such as for nonlinear free-delay systems [1, 2, 27, 25], for nonlinear systems with unknown, time-varying [19, 14, 12]. A reduced-order observer design method is presented in 27] for a class of Lipschitz nonlinear continuous-time systems without time delays which extend the results in 25].

However, in practice, dynamics, measurement, noises or disturbances often prevent the error signals from tending to the origin. Thus, the origin is not a point of equilibrium of the system. An additive term on the right-hand side of the nonlinear system is used to present the uncertainties systems. For this reason, the property is referred to as practical stability which is more suitable for nonlinear free-delay systems ( see [5, 7]) and for nonlinear systems with time-delay ( see [10, 14, 19, 24]). Under unknown, bounded timedelay, an observer design for a class of nonlinear system is presented in [19]. 10] concluded that a class of nonlinear time delay systems is conformed due to some assumptions and the time varying delay bounded the practical exponential stability. Based on conditions in terms of Ricatti differential equation, the problem of 
the practical exponential stability of a class of delayed nonlinear systems is proved in [20] 21 investigated the problem of design for a class of nonlinear systems under unknown time-varying delay. Based on sufficient assumptions, the practical and the exponential stability is achieved.

The main aim of the current paper is to generalize the idea investigated, for instance, in [14, 19] for the purpose of establishing the design of observer. We investigate the problem of exponential convergence of the observation error of a class of nonlinear time-delay systems with constant delay. We impose a generalized condition on the nonlinearity to cover the time-delay systems considered in [3] and a class of systems considered in [14] and [19]. Under unknown and variable time delay and by constructing LyapunovKrasovskii functionals, new criteria are given to insure the practical stability in which the error converges to a small ball.

The rest of this paper is organized as follows: In Section 2, the exponential stable and the practical stability definition are presented and the system description is given. The observer design synthesis method and its stability analysis for a class of nonlinear systems are proved in section 3 . In section 4 , we illustrate our results by a physical model.

\section{System description and basic results}

Consider time delay system of the form:

$$
\left\{\begin{array}{l}
\dot{x}(t)=f(t, x(t), x(t-\tau(t))) \\
x(s)=\varphi(s)
\end{array}\right.
$$

where $\tau(t)$ represents a positive real-value unknown function that denotes the time varying delay affecting both state and input of the system, $x(t, \varphi)$ is the solution of the system with initial function $\varphi$, verifying:

$$
x(s, \varphi)=\varphi(s), \quad \forall s \in[-\tau ; 0] .
$$

$\varphi \in \mathcal{C}$ where $\mathcal{C}$ denotes the Banach space of continuous functions mapping the interval $[-\tau, 0] \rightarrow \mathbb{R}^{n}$ equipped with the supremum-norm:

$$
\|\varphi\|_{\infty}=\max _{s \in[-\tau, 0]}\|\varphi(s)\|
$$

\| $\|$ being the Euclidean-norm. The map $f: \mathbb{R}_{+} \times \mathbb{R}^{n} \times \mathbb{R}^{n} \rightarrow \mathbb{R}^{n}$ is a piecewise continuous function in $t$, and locally Lipschitz in $x$, and satisfies $f(t, 0,0)=0, \forall t \geq 0$.

For $r>0$, denote $B_{r}=\left\{x \in \mathbb{R}^{n} /\|x\| \leq r\right\}$. In the case when $f(t, 0,0) \neq 0$, for certain $t \geq 0$, we shall study the problem of asymptotic stability not for the origin but for a neighborhood of the origin approximated by a small ball of radius $r>0$ centrad at the origin.

The function segment $x_{t}$ is defined by $x_{t}(\theta)=x(t+\theta), \theta \in[-\tau, 0]$. For $\varphi \in \mathcal{C}$, we denote by $x(t, \varphi)$ or shortly $x(t)$ the solution of (11) that satisfies $x_{0}=\varphi$. The segment of this solution is denoted by $x_{t}(\varphi)$ or shortly $x_{t}$.

Definition 1 [22] The zero solution of system (11) is said to be globally exponentially stable with a decay rate $\alpha>0$, if there exist positive reals $\alpha$ and $\beta$ such that, for all $t \geq t_{0}$ and $\varphi \in \mathcal{C}$, the following inequality holds:

$$
\|x(t)\| \leq \beta\|\varphi\|_{\infty} \exp \left(-\alpha\left(t-t_{0}\right)\right) .
$$

Definition 2 [10] We say that $B_{r}$ is globally uniformly exponentially stable if there exist $\lambda_{1}>0$ and $\lambda_{2}>0$ such that for all $t \geq t_{0}$ and $\varphi \in \mathcal{C}$, we have

$$
\|x(t)\| \leq r+\lambda_{1}\|\varphi\|_{\infty} \exp \left(-\lambda_{2}\left(t-t_{0}\right)\right) .
$$

System (11) is globally uniformly practically exponentially stable if there exists $r>0$ such that $B_{r}$ is globally uniformly exponentially stable. 
Remark 1 When $r=0$, in this case the origin is an equilibrium point, then we point the classical definition of the exponential stability (see[4], [22]).

Remark 2 The global uniform practical asymptotic stability of a ball $B_{r}$ defined in this paper is less restrictive than the stability of compact set given in [17] of free-delay systems.

In this paper, we consider the time delay nonlinear system

$$
\left\{\begin{array}{l}
\dot{x}(t)=A x(t)+f(x(t), x(t-\tau(t)), u(t), u(t-\tau(t))), \quad t \geq 0 \\
y(t)=C x(t) \\
x(s)=\varphi(s), \quad \forall s \in[-\tau, 0]
\end{array},\right.
$$

where $x(t) \in \mathbb{R}^{n}$ is the state vector, $u(t) \in \mathbb{R}^{m}$ is the input of the system, $y(t) \in \mathbb{R}$ is the measured output, $\tau(t)$ is a continuously differentiable function which denotes the time-varying delay, $x(t-\tau(t))$ and $u(t-\tau(t))$ are, respectively, the delayed state and input. The matrices $A$, and $C$ are given by,

$$
A=\left[\begin{array}{ccccc}
0 & 1 & 0 & \cdots & 0 \\
0 & 0 & 1 & \cdots & 0 \\
\vdots & \vdots & \vdots & \ddots & \vdots \\
0 & 0 & 0 & \cdots & 1 \\
0 & 0 & 0 & \cdots & 0
\end{array}\right], C=\left[\begin{array}{ccccc}
1 & 0 & \cdots & 0 & 0
\end{array}\right]
$$

and the perturbed term is

$f\left(x(t), x(t-\tau(t), u(t), u(t-\tau(t)))=\left[f_{1}(x(t), x(t-\tau(t)), u(t), u(t-\tau(t))), \cdots, f_{n}(x(t), x(t-\tau(t)), u(t), u(t-\tau(t)))\right]^{T}\right.$.

The mappings $f_{i}: \mathbb{R}^{n} \times \mathbb{R}^{n} \times \mathbb{R}^{m} \times \mathbb{R}^{m} \rightarrow \mathbb{R}, i=1, \ldots, n$, are smooth with $f_{i}(0,0,0,0)=0$.

Throughout the paper, the time argument is omitted and the delayed state vector $x(t-\tau(t))$ is noted by $x^{\tau(t)}$. $A^{T}$ means the transpose of $A . \lambda_{\max }(A)$ and $\lambda_{\min }(A)$ denote the maximal and minimal eigenvalue of a matrix $A$ respectively.

\section{Main results}

We suppose that $f$ satisfies the following assumption:

A1. There exists functions $\gamma_{1}(\varepsilon)>0, \gamma_{2}(\varepsilon)>0$ and $\gamma_{3}(\varepsilon)>0$ such that for $\varepsilon>0$,

$$
\begin{aligned}
\sum_{i=1}^{n} \varepsilon^{i-1}\left|f_{i}\left(x, \bar{x}, u, u^{\tau(t)}\right)-f_{i}\left(y, \bar{y}, u, u^{\tau(t)}\right)\right| & \leq \gamma_{1}(\varepsilon) \sum_{i=1}^{n} \varepsilon^{i-1}\left|x_{i}-y_{i}\right|+\gamma_{2}(\varepsilon) \sum_{i=1}^{n} \varepsilon^{i-1}\left|\bar{x}_{i}-\bar{y}_{i}\right|, \\
\sum_{i=1}^{n} \varepsilon^{i-1}\left|f_{i}\left(x, \bar{x}, u, u^{\tau(t)}\right)-f_{i}\left(x, \bar{y}, u, \bar{u}^{\tau(t)}\right)\right| & \leq \gamma_{2}(\varepsilon) \sum_{i=1}^{n} \varepsilon^{i-1}\left|\bar{x}_{i}-\bar{y}_{i}\right|+\gamma_{3}(\varepsilon) \sum_{i=1}^{n} \varepsilon^{i-1}\left(\left\|u^{\tau(t)}-\bar{u}^{\tau(t)}\right\|\right)(4)
\end{aligned}
$$

Remark 3 We can easily show if the system (2) has a triangular structure (see [15], [14]), that is each $f_{i}$ depends only on $\left(x_{1}, \cdots, x_{i}, x_{1}^{\tau}, \cdots, x_{i}^{\tau}, u, u^{\tau(t)}\right)$ and if we suppose that $f_{i}$ is globally Lipschitz with respect to $\left(x_{1}, \cdots, x_{i}\right),\left(x_{1}^{\tau}, \cdots, x_{i}^{\tau}\right)$ and $u^{\tau(t)}$, uniformly with respect to $u$, which implies that

$$
\begin{aligned}
\left|f_{i}\left(x, \bar{x}, u, u^{\tau(t)}\right)-f_{i}\left(y, \bar{y}, u, \bar{u}^{\tau(t)}\right)\right| \leq & \left|f_{i}\left(x, \bar{x}, u, u^{\tau(t)}\right)-f_{i}\left(x, \bar{z}, u, \bar{u}^{\tau(t)}\right)\right|+\left|f_{i}\left(x, \bar{z}, u, \bar{u}^{\tau(t)}\right)-f_{i}\left(y, \bar{y}, u, \bar{u}^{\tau(t)}\right)\right| \\
\leq & k_{1} \sum_{j=1}^{i}\left(\left|\bar{x}_{j}-\bar{z}_{j}\right|\right)+k_{1}\left\|u^{\tau(t)}-\bar{u}^{\tau(t)}\right\| \\
& +k_{2} \sum_{j=1}^{i}\left(\left|x_{j}-y_{j}\right|+\left|\bar{z}_{j}-\bar{y}_{j}\right|\right)
\end{aligned}
$$


where $k_{1}>0,\left(k_{2}>0\right)$ is a Lipschitz constant in (5), and in (6) respectively, then assumption A1 is fulfilled. Indeed,

$$
\begin{aligned}
\sum_{i=1}^{n} \varepsilon^{i-1}\left|f_{i}\left(x, \bar{x}, u, u^{\tau(t)}\right)-f_{i}\left(y, \bar{y}, u, \bar{u}^{\tau(t)}\right)\right| \leq & \sum_{i=1}^{n} \varepsilon^{i-1} k_{1} \sum_{j=1}^{i}\left(\left|\bar{x}_{j}-\bar{z}_{j}\right|\right)+\sum_{i=1}^{n} \varepsilon^{i-1} k_{1}\left\|u^{\tau(t)}-\bar{u}^{\tau(t)}\right\| \\
& +\sum_{i=1}^{n} \varepsilon^{i-1} k_{2} \sum_{j=1}^{i}\left(\left|x_{j}-y_{j}\right|+\left|\bar{z}_{j}-\bar{y}_{j}\right|\right)
\end{aligned}
$$

But, on the one hand, we have

$$
\begin{aligned}
\sum_{i=1}^{n} \varepsilon^{i-1} k_{2} \sum_{j=1}^{i}\left(\left|x_{j}-y_{j}\right|=\right. & k_{2}\left(1+\varepsilon+\cdots+\varepsilon^{n-1}\right)\left|x_{1}-y_{1}\right| \\
& +k_{2}\left(\varepsilon+\cdots+\varepsilon^{n-1}\right)\left|x_{2}-y_{2}\right|+\cdots+k_{2} \varepsilon^{n-1}\left|x_{n}-y_{n}\right| \\
= & k_{2}\left(1+\varepsilon+\cdots+\varepsilon^{n-1}\right)\left|x_{1}-y_{1}\right| \\
& +k_{2} \varepsilon\left(1+\cdots+\varepsilon^{n-2}\right)\left|x_{2}-y_{2}\right|+\cdots+k_{2} \varepsilon^{n-1}\left|x_{n}-y_{n}\right| \\
\leq & k_{2}\left(1+\varepsilon+\cdots+\varepsilon^{n-1}\right) \sum_{i=1}^{n} \varepsilon^{i-1}\left|x_{i}-y_{i}\right|,
\end{aligned}
$$

and thus also

$$
\begin{aligned}
& \sum_{i=1}^{n} \varepsilon^{i-1} k_{2} \sum_{j=1}^{i}\left(\left|\bar{z}_{j}-\bar{y}_{j}\right| \leq k_{2}\left(1+\varepsilon+\cdots+\varepsilon^{n-1}\right) \sum_{i=1}^{n} \varepsilon^{i-1}\left|\bar{x}_{i}-\bar{y}_{i}\right|,\right. \\
& \sum_{i=1}^{n} \varepsilon^{i-1} k_{1} \sum_{j=1}^{i}\left(\left|\bar{x}_{j}-\bar{y}_{j}\right| \leq k_{1}\left(1+\varepsilon+\cdots+\varepsilon^{n-1}\right) \sum_{i=1}^{n} \varepsilon^{i-1}\left|\bar{x}_{i}-\bar{y}_{i}\right| .\right.
\end{aligned}
$$

and on the other hand, we have

$$
\sum_{i=1}^{n} \varepsilon^{i-1} k_{1}\left\|u^{\tau(t)}-\bar{u}^{\tau(t)}\right\| \leq k_{1}\left(1+\varepsilon+\cdots+\varepsilon^{n-1}\right) \sum_{i=1}^{n} \varepsilon^{i-1}\left\|u^{\tau(t)}-\bar{u}^{\tau(t)}\right\| .
$$

So assumption $\boldsymbol{A} 1$. is satisfied with

$$
\gamma_{1}(\varepsilon)=\gamma_{2}(\varepsilon)=\gamma_{3}(\varepsilon)=k\left(1+\varepsilon+\cdots+\varepsilon^{n-1}\right),
$$

where $k=\max \left(k_{1}, k_{2}\right)$.

Remark 4 In the paper, we deals with the more general systems where the nonlinear function is not necessarily Lipschitz.

\subsection{Observer design}

A tenth of researchers has studied the design problem of the observer for example in [13], 15], 19] and references therein. For nonlinear systems having triangular structures, the global asymptotic stability is proved using a high-gain parameterized linear controller in [15. Under some condition, [13] investigated the problem for exponential observation for nonlinear delay systems. [19] presented observer design for a class of nonlinear system with bounded time-varying delay.

To complete the description of system (2), the following assumption is considered.

A2 For $t \geq 0$, the time delay $\tau(t)=\tau$ is known and constant. 
In this subsection, under constant and known time delay, we present delay-independent conditions to ensure exponential convergence of the observation error. To define the nonlinear time-delay observer for system (2) under assumptions A1 and A2, The following state observer is proposed:

$$
\left\{\begin{array}{l}
\dot{\hat{x}}(t)=A \hat{x}(t)+f\left(\hat{x}(t), \hat{x}^{\tau}, u(t), u^{\tau}\right)+L(\varepsilon)(C \hat{x}(t)-y(t)), \\
\hat{y}(t)=C \hat{x}(t)
\end{array}\right.
$$

where $L(\varepsilon)=\left[\frac{l_{1}}{\varepsilon}, \ldots, \frac{l_{n}}{\varepsilon^{n}}\right]^{T}$ and $L=\left[l_{1}, \ldots, l_{n}\right]^{T}$ such that $A_{L}:=A+L C$ is Hurwitz. Let $P$ be the symmetric positive definite solution of the Lyapunov equation

$$
A_{L}^{T} P+P A_{L}=-I
$$

Theorem 1 Consider the time-delay system (2) under assumptions A1. and A2.. Suppose that there exists $\varepsilon>0$ such that

$$
\frac{\lambda_{\min }(P)}{\varepsilon\|P\|}-2 n \gamma_{1}(\varepsilon)\|P\|-n^{2} \gamma_{2}^{2}(\varepsilon)\|P\|^{2}-1>0
$$

Then, system (7) is a globally exponential observer for system (2).

Proof. Denote $e=\hat{x}-x$ the observation error. We have

$$
\dot{e}=(A+L(\varepsilon) C) e+f\left(\hat{x}, \hat{x}^{\tau}, u, u^{\tau}\right)-f\left(x, x^{\tau}, u, u^{\tau}\right)
$$

For $\varepsilon>0$, let $D(\varepsilon)=\operatorname{diag}\left[1, \varepsilon, \ldots, \varepsilon^{n-1}\right]$. Let $\eta=D(\varepsilon) e$. Using the fact that $A+L(\varepsilon) C=\frac{1}{\varepsilon} D(\varepsilon)^{-1} A_{L} D(\varepsilon)$, we get

$$
\dot{\eta}=\frac{1}{\varepsilon} A_{L} \eta+D(\varepsilon)\left(f\left(\hat{x}, \hat{x}^{\tau}, u, u^{\tau}\right)-f\left(x, x^{\tau} u, u^{\tau}\right)\right)
$$

Let us choose a Lyapunov-Krasovskii functional candidate as follows

$$
V\left(\eta_{t}\right)=V_{1}\left(\eta_{t}\right)+V_{2}\left(\eta_{t}\right)
$$

with

$$
V_{1}\left(\eta_{t}\right)=\eta^{T} P \eta
$$

and

$$
V_{2}\left(\eta_{t}\right)=\int_{t-\tau}^{t} e^{\frac{\sigma}{\tau}(s-t)}\|\eta(s)\|^{2} d s
$$

with $\sigma$ a positive constant defined thereafter.

The time derivative of $V_{1}\left(\eta_{t}\right)$ along the trajectories of system (11) is

$$
\dot{V}_{1}\left(\eta_{t}\right)=\frac{1}{\varepsilon} \eta^{T}\left(A_{L}^{T} P+P A_{L}\right) \eta+2 \eta^{T} P D(\varepsilon)\left(f\left(\hat{x}, \hat{x}^{\tau}, u, u^{\tau}\right)-f\left(x, x^{\tau} u, u^{\tau}\right)\right)
$$

The time derivative of $V_{2}\left(\eta_{t}\right)$ along the trajectories of system (11) is

$$
\dot{V}_{2}\left(\eta_{t}\right)=\|\eta\|^{2}-e^{-\sigma}\left\|\eta^{\tau}\right\|^{2}-\frac{\sigma}{\tau} \int_{t-\tau}^{t} e^{\frac{\sigma}{\tau}(s-t)}\|\eta(s)\|^{2} d s
$$

Since $P$ is symmetric positive definite, for all $\eta \in \mathbb{R}^{n}$,

$$
\lambda_{\min }(P)\|\eta\|^{2} \leq \eta^{T} P \eta \leq \lambda_{\max }(P)\|\eta\|^{2}
$$

Taking the time derivative of (12) along the trajectories of (11), and making use of (8) and (13), we have

$$
\begin{aligned}
\dot{V}\left(\eta_{t}\right) \leq & -\frac{1}{\varepsilon\|P\|} V_{1}(\eta)+2\|\eta\|\|P\|\left\|D(\varepsilon)\left(f\left(\hat{x}, \hat{x}^{\tau}, u, u^{\tau}\right)-f\left(x, x^{\tau} u, u^{\tau}\right)\right)\right\| \\
& +\|\eta\|^{2}-e^{-\sigma}\left\|\eta^{\tau}\right\|^{2}-\frac{\sigma}{\tau} V_{2}(\eta) .
\end{aligned}
$$

Now

$$
\left\|D(\varepsilon)\left(f\left(\hat{x}, \hat{x}^{\tau}, u, u^{\tau}\right)-f\left(x, x^{\tau} u, u^{\tau}\right)\right)\right\| \leq \sum_{i=1}^{n} \varepsilon^{i-1}\left|f_{i}\left(\hat{x}, \hat{x}^{\tau}, u, u^{\tau}\right)-f_{i}\left(x, x^{\tau}, u, u^{\tau}\right)\right|
$$


So using assumption A1. equation(3), we get

$$
\begin{aligned}
\left\|D(\varepsilon)\left(f\left(\hat{x}, \hat{x}^{\tau}, u, u^{\tau}\right)-f\left(x, x^{\tau} u, u^{\tau}\right)\right)\right\| & \leq \gamma_{1}(\varepsilon) \sum_{i=1}^{n} \varepsilon^{i-1}\left|\hat{x}_{i}-x_{i}\right|+\gamma_{2}(\varepsilon) \sum_{i=1}^{n} \varepsilon^{i-1}\left|\hat{x}_{i}^{\tau}-x_{i}^{\tau}\right| \\
& \leq n \gamma_{1}(\varepsilon)\|D(\varepsilon) e\|+n \gamma_{2}(\varepsilon)\left\|D(\varepsilon) e^{\tau}\right\| .
\end{aligned}
$$

Thus

$$
\left\|D(\varepsilon)\left(f\left(\hat{x}, \hat{x}^{\tau}, u, u^{\tau}\right)-f\left(x, x^{\tau} u, u^{\tau}\right)\right)\right\| \leq n \gamma_{1}(\varepsilon)\|\eta\|+n \gamma_{2}(\varepsilon)\left\|\eta^{\tau}\right\|
$$

Thus, we have that

$$
\begin{aligned}
\dot{V}\left(\eta_{t}\right)+\frac{\sigma}{\tau} V\left(\eta_{t}\right) \leq & -\left(\frac{1}{\varepsilon\|P\|}-\frac{\sigma}{\tau}\right) V_{1}(\eta)+2 n \gamma_{1}(\varepsilon)\|P\|\|\eta\|^{2} \\
& +2 n \gamma_{2}(\varepsilon)\|P\|\|\eta\|\left\|\eta^{\tau}\right\|+\|\eta\|^{2}-e^{-\sigma}\left\|\eta^{\tau}\right\|^{2} \\
\leq & -\left\{\lambda_{\min }(P)\left(\frac{1}{\varepsilon\|P\|}-\frac{\sigma}{\tau}\right)-2 n \gamma_{1}(\varepsilon)\|P\|-1\right\}\|\eta\|^{2} \\
& +2 n \gamma_{2}(\varepsilon)\|P\|\|\eta\|\left\|\eta^{\tau}\right\|-e^{-\sigma}\left\|\eta^{\tau}\right\|^{2}
\end{aligned}
$$

Hence, we have that

$$
\dot{V}\left(\eta_{t}\right)+\frac{\sigma}{\tau} V\left(\eta_{t}\right) \leq-a(\sigma, \varepsilon)\|\eta\|^{2}+b(\varepsilon)\|\eta\|\left\|\eta^{\tau}\right\|-e^{-\sigma}\left\|\eta^{\tau}\right\|^{2}
$$

with

$$
\begin{aligned}
a(\sigma, \varepsilon) & =\lambda_{\min }(P)\left(\frac{1}{\varepsilon\|P\|}-\frac{\sigma}{\tau}\right)-2 n \gamma_{1}(\varepsilon)\|P\|-1, \\
b(\varepsilon) & =2 n \gamma_{2}(\varepsilon)\|P\| .
\end{aligned}
$$

Now, the right side of the inequality (16) can be rewritten as follows

$$
-a(\sigma, \varepsilon)\|\eta\|^{2}+b(\varepsilon)\|\eta\|\left\|\eta^{\tau}\right\|-e^{-\sigma}\left\|\eta^{\tau}\right\|^{2}=-\left(a(\sigma, \varepsilon)-\frac{b^{2}(\varepsilon)}{4} e^{\sigma}\right)\|\eta\|^{2}-\left(\frac{b(\varepsilon)}{2} e^{\frac{\sigma}{2}}\|\eta\|-e^{-\frac{\sigma}{2}}\left\|\eta^{\tau}\right\|\right)^{2}
$$

To satisfy inequality (16), all we need to do is to choose $\sigma$ such that

$$
\left(a(\sigma, \varepsilon)-\frac{b^{2}(\varepsilon)}{4} e^{\sigma}\right)>0,
$$

which is equivalent to

$$
\lambda_{\min }(P) \frac{\sigma}{\tau}+\frac{b^{2}(\varepsilon)}{4}\left(e^{\sigma}-1\right)<\frac{\lambda_{\min }(P)}{\varepsilon\|P\|}-2 n \gamma_{1}(\varepsilon)\|P\|-1-\frac{b^{2}(\varepsilon)}{4}
$$

Let $w(x)=\lambda_{\min }(P) \frac{x}{\tau}+\frac{b^{2}(\varepsilon)}{4}\left(e^{x}-1\right)$, we have $w(x)>0 \forall x>0$ and $w(0)=0$.

Since $w$ is continuous at 0 , there exists $\delta>0$ such that $\forall x \in] 0, \delta\left[, 0<w(x)<\frac{\lambda_{\min }(P)}{\varepsilon\|P\|}-2 n \gamma_{1}(\varepsilon)\|P\|-\frac{4+b^{2}(\varepsilon)}{4}\right.$. Let $\sigma \in] 0, \delta[$, then inequality (17) is verified.

Now, the objective is to prove the exponential convergence of the observer (10).

Using (9), inequality (16) becomes

$$
\dot{V}\left(\eta_{t}\right) \leq-\frac{\sigma}{\tau} V\left(\eta_{t}\right)
$$

It follows that

$$
V\left(\eta_{t}\right) \leq e^{-\frac{\sigma}{\tau} t} V\left(\eta_{t}(0)\right)
$$

Using (12) and (13), we have, on the one hand,

$$
\begin{aligned}
V\left(\eta_{t}(0)\right) & \leq \lambda_{\max }(P)\left\|\eta_{t}(0)\right\|^{2}+\int_{-\tau}^{0} e^{\frac{\sigma}{\tau} s}\|\eta(s)\|^{2} d s \\
& \leq\left(\lambda_{\max }(P)+\tau\right) \sup _{s \in[-\tau, 0]}\|\eta(s)\|^{2}
\end{aligned}
$$


and on the other hand,

$$
\lambda_{\min }(P)\left\|\eta_{t}\right\|^{2} \leq V\left(\eta_{t}\right)
$$

We deduce that

$$
\left\|\eta_{t}\right\| \leq \sqrt{\frac{\|P\|+\tau}{\lambda_{\min }(P)}} e^{\frac{-\sigma}{2 \tau} t} \sup _{s \in[-\tau, 0]}\|\eta(s)\| .
$$

Finally, with $\eta=D(\varepsilon) e$, the observation error $e(t)$ is given by

$$
\|e(t)\| \leq \frac{1}{\|D(\varepsilon)\|} \sqrt{\frac{\|P\|+\tau}{\lambda_{\min }(P)}} e^{\frac{-\sigma}{2 \tau} t} \sup _{s \in[-\tau, 0]}\|\eta(s)\| .
$$

Then, the error dynamics (10) is globally exponentially stable.

Remark 5 In [26], based on linear matrix inequalities, the authors developed the sufficient conditions which guarantee the estimation error converge asymptotically towards zero. As compared to [26], our results are less conservative and more convenient to use since they are independent of time delays.

Remark 6 [8], proposed a state feedback controller that are synthesized under sufficient conditions expressed in terms of Riccati differential equations and linear matrix inequalities which can stabilize the studied nonlinear uncertain systems with time-varying delay. Feedback controllers are synthesized under sufficient conditions linear matrix inequalities and expressed in terms of Riccati differential equations. But, in this paper, we use a parameter in order to establish global asymptotical stability of the nonlinear system.

\subsection{Practical exponential stability}

In this section, we give sufficient conditions to ensure the practical stability convergence. In fact, in the real world, the problem of practical stability is more appropriate. Then, for practical purpose, practical stability seems desirable ( see [5] ) for systems without delays and [24, [10] and [14] for time-delay systems. In the general case, one can not directly measure the states of a system. Thus, one must observe the unmeasured states. An observer is a dynamical system which estimates the states of the system.

In this section, for complete the description of system (2) the following assumptions are needed.

H1. The state and the input are considered bounded, that is $x(t) \in K \subset \mathbb{R}^{n}$ ( that is a compact subset of $\left.\mathbb{R}^{n}\right)$.

H2. The time-varying delay satisfies the following properties:

(i) $\exists \tau^{*}>0$, such that $0 \leq \tau(t) \leq \tau^{*}$.

(ii) $\exists \beta>0$, such that $\dot{\tau}(t) \leq 1-\beta$.

Remark 7 The boundedness of the state excludes implicitly all initial conditions that generate unbounded state.

The following state observer for system (2) under assumption H1., H2. and A1. is proposed:

$$
\left\{\begin{array}{l}
\dot{\hat{x}}(t)=A \hat{x}(t)+f\left(\hat{x}(t), \hat{x}^{\tau^{*}}, u(t), u^{\tau^{*}}\right)+L(\varepsilon)(C \hat{x}(t)-y(t)), \\
\hat{y}(t)=C \hat{x}(t),
\end{array}\right.
$$

Let us now define $e=\hat{x}-x$ the observation error, which denotes the difference between the actual state and estimated states.

Theorem 2 Consider the time-delay system (2) under assumptions H1., H2. and A1.. Suppose that there exists $\varepsilon>0$ such that

$$
\frac{\lambda_{\min }(P)}{\varepsilon\|P\|}-2 n \gamma_{1}(\varepsilon)\|P\|-\frac{5}{4}-n^{2} \gamma_{2}^{2}(\varepsilon)\|P\|^{2}>0
$$

Then, the error dynamics (21) is globally (on $K$ ) practically exponentially stable. 
Proof. We have

$$
\dot{e}=(A+L(\varepsilon) C) e+f\left(\hat{x}, \hat{x}^{\tau^{*}}, u, u^{\tau^{*}}\right)-f\left(x, x^{\tau(t)}, u, u^{\tau(t)}\right)
$$

For $\varepsilon>0$, let $D(\varepsilon)=\operatorname{diag}\left[1, \varepsilon, \ldots, \varepsilon^{n-1}\right]$. Let $\eta=D(\varepsilon) e$. Using the fact that $A+L(\varepsilon) C=\frac{1}{\varepsilon} D(\varepsilon)^{-1} A_{L} D(\varepsilon)$, we get

$$
\dot{\eta}=\frac{1}{\varepsilon} A_{L} \eta+D(\varepsilon)\left(f\left(\hat{x}, \hat{x}^{\tau^{*}}, u, u^{\tau^{*}}\right)-f\left(x, x^{\tau(t)}, u, u^{\tau(t)}\right)\right)
$$

Let us choose a Lyapunov-Krasovskii functional candidate as follows

$$
W\left(t, \eta_{t}\right)=\eta^{T} P \eta+\int_{t-\tau(t)}^{t} e^{\frac{\sigma}{\tau^{*}(s-t)}}\|\eta(s)\|^{2} d s
$$

where $P$ is provided by (8) and $\sigma$ a positive constant defined thereafter.

The time derivative of $W\left(t, \eta_{t}\right)$ along the trajectories of system (22) is

$$
\begin{aligned}
\dot{W}\left(t, \eta_{t}\right)= & \frac{1}{\varepsilon} \eta^{T}\left(A_{L}^{T} P+P A_{L}\right) \eta+2 \eta^{T} P D(\varepsilon)\left(f\left(\hat{x}, \hat{x}^{\tau^{*}}, u, u^{\tau^{*}}\right)-f\left(x, x^{\tau(t)}, u, u^{\tau(t)}\right)\right) \\
& +\|\eta\|^{2}-(1-\dot{\tau}(t)) e^{-\frac{\sigma \tau(t)}{\tau^{*}}}\left\|\eta^{\tau}\right\|^{2}-\frac{\sigma}{\tau} \int_{t-\tau(t)}^{t} e^{\frac{\sigma}{\tau^{*}}(s-t)}\|\eta(s)\|^{2} d s .
\end{aligned}
$$

As in the proof of Theorem 1 and using (8), (13) and assumption H2., we have

$$
\begin{aligned}
\dot{W}\left(t, \eta_{t}\right)+\frac{\sigma}{\tau^{*}} W\left(t, \eta_{t}\right) \leq & -\left\{\lambda_{\min }(P)\left(\frac{1}{\varepsilon\|P\|}-\frac{\sigma}{\tau^{*}}\right)-1\right\}\|\eta\|^{2}-\beta e^{-\sigma}\left\|\eta^{\tau^{*}}\right\|^{2} \\
& +2\|\eta\|\|P\|\left\|D(\varepsilon)\left(f\left(\hat{x}, x^{\tau^{*}}, u, u^{\tau^{*}}\right)-f\left(x, x^{\tau(t)}, u, u^{\tau(t)}\right)\right)\right\| .
\end{aligned}
$$

Now, the majorization of the term $\left\|D(\varepsilon)\left(f\left(\hat{x}, \hat{x}^{\tau^{*}}, u, u^{\tau^{*}}\right)-f\left(x, x^{\tau(t)}, u, u^{\tau(t)}\right)\right)\right\|$. Characterizes the difference between the term that depends on the upper bound of the unknown delay and the term which depends on the unknown delay.

$$
\begin{aligned}
\left\|D(\varepsilon)\left(f\left(\hat{x}, \hat{x}^{\tau^{*}}, u, u^{\tau^{*}}\right)-f\left(x, x^{\tau(t)}, u, u^{\tau(t)}\right)\right)\right\| & \leq\left\|D(\varepsilon)\left(f\left(\hat{x}, \hat{x}^{\tau^{*}}, u, u^{\tau^{*}}\right)-f\left(x, x^{\tau^{*}}, u, u^{\tau^{*}}\right)\right)\right\| \\
& +\left\|D(\varepsilon)\left(f\left(x, x^{\tau^{*}}, u, u^{\tau^{*}}\right)-f\left(x, x^{\tau(t)}, u, u^{\tau(t)}\right)\right)\right\| .
\end{aligned}
$$

Using (14) and assumption A1., on the one hand, we get

$$
\begin{aligned}
\left\|D(\varepsilon)\left(f\left(\hat{x}, \hat{x}^{\tau^{*}}, u, u^{\tau^{*}}\right)-f\left(x, x^{\tau^{*}}, u, u^{\tau^{*}}\right)\right)\right\| & \leq \gamma_{1}(\varepsilon) \sum_{i=1}^{n} \varepsilon^{i-1}\left|\hat{x}_{i}-x_{i}\right|+\gamma_{2}(\varepsilon) \sum_{i=1}^{n} \varepsilon^{i-1}\left|\hat{x}_{i}^{\tau^{*}}-x_{i}^{\tau^{*}}\right| \\
& \leq n \gamma_{1}(\varepsilon)\|D(\varepsilon) e\|+n \gamma_{2}(\varepsilon)\left\|D(\varepsilon) e^{\tau^{*}}\right\| .
\end{aligned}
$$

Thus

$$
\left\|D(\varepsilon)\left(f\left(\hat{x}, \hat{x}^{\tau^{*}}, u, u^{\tau^{*}}\right)-f\left(x, x^{\tau^{*}}, u, u^{\tau^{*}}\right)\right)\right\| \leq n \gamma_{1}(\varepsilon)\|\eta\|+n \gamma_{2}(\varepsilon)\left\|\eta^{\tau^{*}}\right\|,
$$

and on the other hand,

$$
\left\|D(\varepsilon)\left(f\left(x, x^{\tau^{*}}, u, u^{\tau^{*}}\right)-f\left(x, x^{\tau(t)}, u, u^{\tau(t)}\right)\right)\right\| \leq \gamma_{2}(\varepsilon) \sum_{i=1}^{n} \varepsilon^{i-1}\left|x_{i}^{\tau^{*}}-x_{i}^{\tau(t)}\right|+\gamma_{3}(\varepsilon) \sum_{i=1}^{n} \varepsilon^{i-1}\left|u_{i}^{\tau^{*}}-u_{i}^{\tau(t)}\right| .
$$

From assumption H1., there exists a bounded constant $\nu_{1}$ and $\nu_{2}$ such that (25) can be written as follows:

$$
\left\|D(\varepsilon)\left(f\left(x, x^{\tau^{*}}, u, u^{\tau^{*}}\right)-f\left(x, x^{\tau(t)}, u, u^{\tau(t)}\right)\right)\right\| \leq \gamma_{2}(\varepsilon) \frac{1-\varepsilon^{n}}{1-\varepsilon} \nu_{1}+\gamma_{3}(\varepsilon) \frac{1-\varepsilon^{n}}{1-\varepsilon} \nu_{2} .
$$

where $\nu_{1}$ and $\nu_{2}$ are respectively, the positive constant which refers to the boundedness of $\left\|x^{\tau^{*}}-x^{\tau(t)}\right\|$ and $\left\|u^{\tau^{*}}-u^{\tau(t)}\right\|$. Let

$$
\theta= \begin{cases}2\|P\|\left(\gamma_{2}(\varepsilon)+\gamma_{3}(\varepsilon)\right) \frac{1-\varepsilon^{n}}{1-\varepsilon} \nu, & \text { if } \varepsilon \neq 1 \\ 2\|P\|\left(\gamma_{2}(\varepsilon)+\gamma_{3}(\varepsilon)\right) n \nu, & \text { if } \varepsilon=1\end{cases}
$$


where $\nu=\max \left(\nu_{1}, \nu_{2}\right)$. Thus

$$
2\|P\|\left\|D(\varepsilon)\left(f\left(x, x^{\tau^{*}}, u, u^{\tau^{*}}\right)-f\left(x, x^{\tau(t)}, u, u^{\tau(t)}\right)\right)\right\| \leq \theta .
$$

Using (24) and (26), we have

$$
\begin{aligned}
\dot{W}\left(t, \eta_{t}\right)+\frac{\sigma}{\tau^{*}} W\left(t, \eta_{t}\right) \leq & -\left\{\lambda_{\min }(P)\left(\frac{1}{\varepsilon\|P\|}-\frac{\sigma}{\tau^{*}}\right)-2 n \gamma_{1}(\varepsilon)\|P\|-1\right\}\|\eta\|^{2} \\
& +\|\eta\| \theta+2 n \gamma_{2}(\varepsilon)\|P\|\|\eta\|\left\|\eta^{\tau^{*}}\right\|-\beta e^{-\sigma}\left\|\eta^{\tau^{*}}\right\|^{2}
\end{aligned}
$$

Using the fact that

$$
\theta\|\eta\| \leq \frac{1}{4}\|\eta\|^{2}+\theta^{2}
$$

we deduce that

$$
\dot{W}\left(t, \eta_{t}\right)+\frac{\sigma}{\tau^{*}} W\left(t, \eta_{t}\right)-\theta^{2} \leq-c(\sigma, \varepsilon)\|\eta\|^{2}+b(\varepsilon)\|\eta\|\left\|\eta^{\tau^{*}}\right\|-\beta e^{-\sigma}\left\|\eta^{\tau^{*}}\right\|^{2}
$$

with

$$
\begin{aligned}
c(\sigma, \varepsilon) & =\lambda \min (P)\left(\frac{1}{\varepsilon\|P\|}-\frac{\sigma}{\tau^{*}}\right)-2 n \gamma_{1}(\varepsilon)\|P\|-\frac{5}{4}, \\
b(\varepsilon) & =2 n \gamma_{2}(\varepsilon)\|P\| .
\end{aligned}
$$

Now, the right side of the inequality (27) can be rewritten as follows

$-c(\sigma, \varepsilon)\|\eta\|^{2}+b(\varepsilon)\|\eta\|\left\|\eta^{\tau^{*}}\right\|-\beta e^{-\sigma}\left\|\eta^{\tau^{*}}\right\|^{2}=-\left(c(\sigma, \varepsilon)-\frac{b^{2}(\varepsilon)}{4 \beta} e^{\sigma}\right)\|\eta\|^{2}-\left(\frac{b(\varepsilon)}{2 \sqrt{\beta}} e^{\frac{\sigma}{2}}\|\eta\|-\sqrt{\beta} e^{-\frac{\sigma}{2}}\left\|\eta^{\tau^{*}}\right\|\right)^{2}$.

To satisfy inequality (27), all we need to do is to choose $\sigma$ such that

$$
\left(c(\sigma, \varepsilon)-\frac{b^{2}(\varepsilon)}{4 \beta} e^{\sigma}\right)>0
$$

which is equivalent to

$$
\lambda_{\min }(P) \frac{\sigma}{\tau^{*}}+\frac{b^{2}(\varepsilon)}{4}\left(e^{\sigma}-1\right)<\frac{\lambda_{\min }(P)}{\varepsilon\|P\|}-2 n \gamma_{1}(\varepsilon)\|P\|-\frac{5}{4}-\frac{b^{2}(\varepsilon)}{4 \beta}
$$

As in the proof of Theorem 1 and let $\sigma \in] 0, \delta[$, then inequality (28) is verified.

Now, the objective is to prove the uniform practical stability of (21).

Using (20), inequality (27) becomes

It follows that

$$
\dot{W}\left(\eta_{t}\right) \leq-\frac{\sigma}{\tau^{*}} W\left(\eta_{t}\right)+\theta^{2}
$$

$$
W\left(\eta_{t}\right) \leq e^{-\frac{\sigma}{\tau^{*} t} W}\left(\eta_{t}(0)\right)+2 \theta^{2} \frac{\tau^{*}}{\sigma}
$$

Using (13), we have, on the one hand,

$$
\begin{aligned}
W\left(\eta_{t}(0)\right) & \leq \lambda_{\max }(P)\left\|\eta_{t}(0)\right\|^{2}+\int_{-\tau^{*}}^{0} e^{\frac{\sigma}{\tau} s}\|\eta(s)\|^{2} d s \\
& \leq\left(\lambda_{\max }(P)+\tau^{*}\right) \sup _{s \in[-\tau, 0]}\|\eta(s)\|^{2}
\end{aligned}
$$

and on the other hand,

$$
\lambda_{\min }(P)\left\|\eta_{t}\right\|^{2} \leq V\left(\eta_{t}\right)
$$

We deduce that

$$
\left\|\eta_{t}\right\| \leq \sqrt{\frac{\|P\|+\tau^{*}}{\lambda_{\min }(P)}} e^{\frac{-\sigma}{2 \tau^{*}} t} \sup _{s \in\left[-\tau^{*}, 0\right]}\|\eta(s)\|+\sqrt{\frac{2 \theta^{2} \tau^{*}}{\sigma \lambda_{\min (P)}}} .
$$

Finally, with $\eta=D(\varepsilon) e$, the observation error $e(t)$ is given by

$$
\|e(t)\| \leq \frac{1}{\|D(\varepsilon)\|} \sqrt{\frac{\|P\|+\tau^{*}}{\lambda_{\min }(P)}} e^{\frac{-\sigma}{2 \tau} t} \sup _{s \in[-\tau, 0]}\|\eta(s)\|+\frac{1}{\|D(\varepsilon)\|} \sqrt{\frac{2 \theta^{2} \tau^{*}}{\sigma \lambda_{\min (P)}}} .
$$

Then, the error dynamics (21) is globally (on K) practically exponentially stable. 
Remark 8 It is noted that if system (2) satisfies condition (6) inspired in [15] and [14], then assumption A1. is satisfied with $\gamma_{1}(\varepsilon)=\gamma_{2}(\varepsilon)=\gamma_{3}(\varepsilon)=k\left(1+\varepsilon+\cdots+\varepsilon^{n-1}\right)$. Let

$$
\begin{aligned}
& c_{1}(\varepsilon)=\frac{\lambda_{\min }(P)}{\varepsilon\|P\|}-2 n \gamma_{1}(\varepsilon)\|P\|-n^{2} \gamma_{2}^{2}(\varepsilon)\|P\|^{2}-1, \\
& c_{2}(\varepsilon)=\frac{\lambda_{\min }(P)}{\varepsilon\|P\|}-2 n \gamma_{1}(\varepsilon)\|P\|-n^{2} \gamma_{2}^{2}(\varepsilon)\|P\|^{2}-\frac{5}{4} .
\end{aligned}
$$

In this case, $c_{1}(\varepsilon)$ and $c_{2}(\varepsilon)$ tend to $\infty$ as $\varepsilon$ tends to zero. This implies that there exists $\varepsilon_{1}>0$ such that for all $0<\varepsilon<\varepsilon_{1}$ conditions (9) and (20) are fulfilled.

\section{Numerical example}

This section presents experimental result, we give an example of the orientational motion of polar molecules acted on by an external perturbation. We consider a physical model corresponding to a slow relaxation process. The dynamics model systems are represented by:

$$
\begin{aligned}
& \dot{x}_{1}=x_{2}+\frac{1}{12} \sin x_{2}(t-\tau(t))+\frac{1}{24} x_{1} \cos (u(t-\tau(t)), \\
& \dot{x}_{2}=x_{3}+\frac{1}{12} x_{2}(t-\tau(t))+\frac{1}{24} x_{2}+u(t-\tau(t)), \\
& y=x_{1} .
\end{aligned}
$$

where the input $u(t)=\cos (7 t)$ denote the orientational potential energy, the function $\tau(t)$ is defined as follows: $\tau(t)=0.25+0.01 \cos ^{2}(t)$ being the Debye relaxation time $x(t)$ is the augmented state vector containing the plant state vector. Following the notation used throughout the paper, let $f_{1}\left(x, x^{\tau(t)}, u, u^{\tau(t)}\right)=$ $\frac{1}{12} \sin x_{2}(t-\tau(t))+\frac{1}{24} x_{1} \cos (u(t-\tau(t))), f_{2}\left(x, x^{\tau(t)}, u, u^{\tau(t)}\right)=\frac{1}{12} x_{2}(t-\tau(t))+\frac{1}{24} x_{2}+u(t-\tau(t)$. Furthermore, since $f_{1}$ depends on $x_{2}^{\tau(t)}$, the method proposed in [14] is not applicable in this case. The input and the states are bounded, which make assumption $\mathrm{H} 1$ holds. It is easy to check that system (30) satisfies Assumption A1 with $\gamma_{1}(\varepsilon)=\frac{1}{24}(1+\varepsilon), \gamma_{2}(\varepsilon)=\frac{1}{12}(1+\varepsilon)$ and $\gamma_{3}(\varepsilon)=(1+\varepsilon)$.

The initial conditions for the system are $x(0)=\left[\begin{array}{ll}-2 & 1\end{array}\right]^{T}$, for the observer $\hat{x}(0)=\left[\begin{array}{ll}2 & 2\end{array}\right]^{T}$. Now, select $L=\left[\begin{array}{ll}-5 & -5\end{array}\right]^{T}$ and $A_{L}$ is Hurwitz. Using Matlab, the solutions of the Lyapunov equations (8) is given by

$$
P=\left[\begin{array}{ll}
0.1200 & 0.1000 \\
0.1000 & 1.1000
\end{array}\right] .
$$

So, $\lambda_{\max }(P)=1.1101$ and $\lambda_{\min }(P)=0.1099$. This implies that condition (20) is satisfied for all $0<\varepsilon<0.06$. According to the practical stability improved in the proof of Theorem 2 it is clear from Figs. 1 and 2 that the global uniform practical convergence is ensured with radius For our numerical simulation, we choose $\varepsilon=0.05$.

\section{Conclusion}

In this paper, the problem of global uniform practical exponential stability and an observer for a class of time-delay nonlinear systems have been considered. This class of systems covers the systems having a triangular structure. In the case of a constant time delay, we have derived delay-independent conditions to ensure global exponential stability. We have suggested sufficient conditions to guarantee a practical stability of the proposed observer in the case of a bounded and unknown variable time delay. Finally, the effectiveness of the conditions obtained in this paper is verified in a numerical example. 


\section{References}

[1] Atassi, A. N., Khalil, H. K.: A separation principle for the stabilization of a class of nonlinear systems. IEEE Transactions on Automatic Control, 44,(1999), 1672-1687.

[2] Atassi, A. N., Khalil, H. K.: Separation results for the stabilization of nonlinear systems using different high-gain observer designs. Systems and Control Letters, 39, (2000), 183-191.

[3] Benabdallah, A.: A separation principle for the stabilization of a class of time delay nonlinear systems. Kybernetika, 51 ( 2015 ), 1, 99-111.

[4] Benabdallah, A., Echi, N. Global exponential stabilisation of a class of nonlinear time-delay systems. International Journal of Systems Science, 47, 3857-3863, (2016).

[5] Benabdallah, A., Ellouze, I., Hammami, M.A. Practical stability of nonlinear time-varying cascade systems. Journal of Dynamical and Control Systems, 15, 45-62, (2009).

[6] Cho, H. Ch., Park, J. H. Stable bilateral teleoperation under a time delay using a robust impedance control. Mechatronics, 15,(5),611-625, (2005).

[7] Corless, M., Guaranteed rates of exponential convergence for uncertain systems. Journal of optimization theory and applications, 64,(1990).

[8] Dong, Y., Wang, X., Mei, S., Li, W. (2012), Exponential stabilization of nonlinear uncertain systems with time-varying delay. Journal of Engineering Mathematics 77, 225-237(2012).

[9] Echi, N., Benabdallah, A. Delay-dependent stabilization of a class of time-delay nonlinear systems: LMI approach. Advances in Difference Equations 2017, 271 (2017).

[10] Ben Hamed, B., Ellouze, I., Hammami, M.A. Practical uniform stability of nonlinear differential delay equation. Mediterranean Journal of Mathematics 8, 603616, (2011).

[11] Ben Hamed, Hammami, M.A. Practical stabilization of a class of uncertain time-varying nonlinear delay systems.Journal of Control Theory and Applications 20097 (2) 175-180.

[12] Farza, M., Sboui, A., Cherrier, E., M'Saad, M. High-gain observer for a class of time-delay nonlinear systems., International Journal of Control, 83, 2, 273-280, (2010).

[13] Germani,A., Manes,C., Pepe, P. An asymptotic state observer for a class of nonlinear delay systems. Kybernetika 37, 459-478, (2001).

[14] Ghanes, M., De Leon, J., Barbot, J. Observer design for nonlinear systems under unknown time-varying delays. IEEE Transactions on Automatic Control 58, 1529-1534 (2013).

[15] Ibrir, S.: Observer-based control of a class of time-delay nonlinear systems having triangular structure. Automatica 47, 388-394, (2011).

[16] Lili, C., Ying, Z., Xian, Z.Guaranteed cost control for uncertain genetic regulatory networks with interval time-varying delays. Neurocomputing 131, 105-112, (2014).

[17] Lin,Y., Sontag E. D., Wang, Y. A smooth converse Lyapunov theorem for robust stability. SIAM J. Control Optim. 34, 124-160(1996).

[18] Muroya, Y., Kuniya, T., Wang, J.L. Stability analysis of a delayed multi-group SIS epidemic model with nonlinear incidence rates and patch structure. Journal of Mathematical Analysis and Applications, 425(1), 415-439, (2015).

[19] Naifar, O., Ben Makhlouf, A., Hammami, M.A., Ouali, A.On Observer Design for a Class of Nonlinear Systems Including Unknown Time-Delay. Mediterranean Journal of Mathematics 13, ( 5), 2841-2851, (2016). 
[20] Naifar, O., Ben Makhlouf, A., Hammami, M.A., Practical stability for a class of nonlinear time varying systems including delayed perturbation. IEEE 10.1109/WSMEAP.2015.7338209(2015).

[21] Naifar, O., Ben Makhlouf, A., Hammami, M.A., Ouali, A.State feedback control law for a class of nonlinear time-varying system under unknown time-varying delay. Nonlinear Dynamics, 82, 12, pp 349355(2015).

[22] Pepe, P., Karafyllis, I. Converse Lyapunov-Krasovskii theorems for systems described by neutral functional differential equations in Hales form. International Journal of Control, 86(2), 232-243, (2013).

[23] Pham,Q. C., Tabareau, N., Slotine, J. E. A contraction theory appoach to stochastic Incremental stability. IEEE Transactions on Automatic Control, 54, 1285-1290, (2009).

[24] Villafuerte, R., Mondie, S., Poznyak, A. Practical Stability of Time-Delay Systems: LMI's Approach. European Journal of Control, 2, 127-138, (2011).

[25] Rajamani, R., Observers for Lipschitz nonlinear systems. IEEE Transactions on Automatic Control, 43, , (1998).

[26] Zhou, L., Xiao, X., Lu, G. Observers for a Class of Nonlinear Systems with Time-Delay. Asian Journal of Control, 11, 688-693,(2009).

[27] Zhu, F., Han, Z., A note on observers for Lipschitz nonlinear systems. IEEE Transactions on Automatic Control, 47, 10, (2002). 